\title{
Study on Small Vibration Sensor by Self-Coupling Effect of Semiconductor Laser
}

\author{
Yasuhiko NAWA, Norio TSUDA and Jun YAMADA \\ Aichi Institute of Technology, 1247 Yachigusa, Yagusa-cho, Toyota, Aichi 470-0392
}

(Received January 28, 2008)

\begin{abstract}
Small vibration sensors with the laser interferometer have been developed. However, there are some disadvantages, for example, weakness to external vibration, difficulty in optical alignment, and so on. These disadvantages are improved by making use of a self-coupling effect of a semiconductor laser, because our sensor is composed of only a laser diode and a lens. When a small vibration, whose amplitude is less than half-wavelength of the laser, is measured, a reference signal is needed. The reference signal is generated by moving the sensor head quickly. And also an output signal is needed at maximum sensitivity. This output signal is generated by moving the sensor head slowly. The signal is controlled by a microcomputer. The vibration amplitude is evaluated from the ratio of the output signal to the reference one by the microcomputer. This sensor can detect a small vibration with minimum amplitude of about $10 \mathrm{~nm}$, and a small vibration is able to be measured irrespective of the frequency in the range between $500 \mathrm{~Hz}$ and $3000 \mathrm{~Hz}$.
\end{abstract}

Key Words: Self-coupling effect, Small vibration, Sensor, Semiconductor laser, Microcomputer

\section{Introduction}

Recently, in development of the IC technology, a line width of IC has been reduced to tens of $\mathrm{nm}$ and demand for compact small vibration sensors in the manufacturing process increases. The laser interferometer is a conventional non-contact method of detecting small vibrations, but this method has some disadvantages, including weakness to external vibration and difficulty in optical alignment. Making use of a self-coupling effect of the laser diode (LD), velocity ${ }^{1,2)}$, vibration ${ }^{3,4)}$ and distance meters $^{5,6)}$ have been studied. They could accurately detect a vibration with large amplitude of more than half-wavelength of the LD. However, the resolution of these sensors is the order of half-wavelength. It is difficult to detect vibrations accurately when the vibration amplitude is less than half-wavelength of the LD. Because the output signal of the sensor varies with not the change in the vibration amplitude of the target but the change in the back-scattered intensity from the target. To avoid these problems, we have proposed a simple and compact sensor with a reference signal, which is proofed against external vibrations and can detect a small vibration by making use of a self-coupling effect of the LD. Then the small vibration amplitude is determined by comparing the output signal to the reference signal. The reference signal is generated by moving the sensor head quickly. This sensor could detect the small vibration with minimum amplitude of about $10 \mathrm{~nm}$ at the frequency between $500 \mathrm{~Hz}$ and $3000 \mathrm{~Hz}$. And also the small vibration frequency between $500 \mathrm{~Hz}$ and $3000 \mathrm{~Hz}$ was able to be measured.

\section{Principle of Measurement}

A schematic diagram for a principle of a self-coupling effect is shown in Fig.1. The laser light from the LD illuminates an outside target, and a fraction of the back-scattered light from the target reenters into the laser cavity. The back-scattered light interferes with the laser light in the laser cavity, and then the output signal slightly changes. This is referred to as a

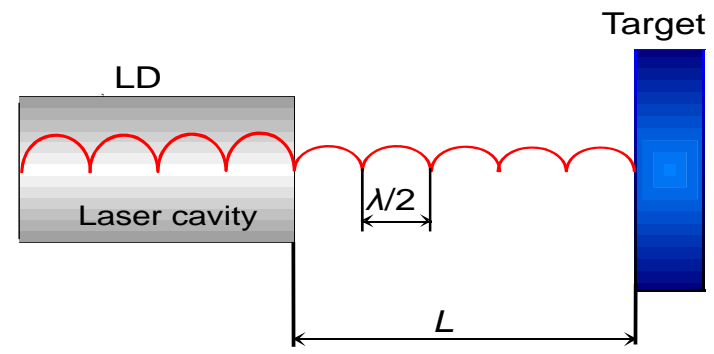

Fig.1 Schematic diagram for self-coupling effect

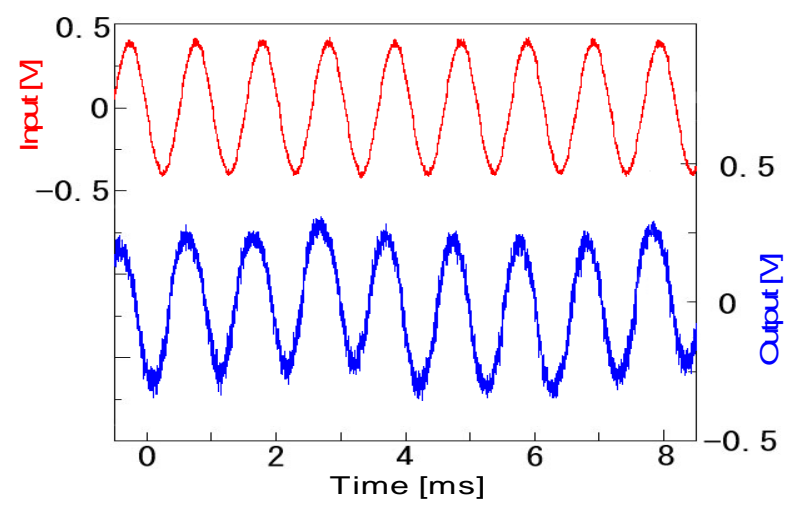

Fig.2 Interference signal with amplitude of less than half-wavelength of LD 
self-coupling effect. When the resonance condition $L=n \lambda / 2$ (where $L$ is the distance between the cleaved surface of the LD and the target, $n$ is integer, and $\lambda$ is the wavelength of the LD) is satisfied, the output light slightly increases.

The measured interference signal of a small vibration and the input vibration signal are shown in Fig.2. When the target vibrates with small amplitude of less than half-wavelength of the LD, whether the cause of the change in interference signal is a change in the back-scattered intensity from the target or in the vibration amplitude of the target can not be distinct. Because the phase between the input signal and the output one is identical or inverse as shown in Fig.2. Then, the sensor head is moved by a distance of more than half-wavelength of the LD and the output signal as a reference is generated. The small vibration amplitude is determined by comparing the output signal by the target vibration to the reference signal.

When the vibration amplitude is less than half-wavelength of the LD, the input signals and the output signals are shown in Fig. 3 for two cases; the vibration amplitude of the target are the same, but the distance between the target and the sensor is different. Even if the vibration amplitude of the target is the same, the different output signals appear. In Fig.3, the upper output signal is twice of the lower one. The sensor sensitivity changes depending on a distance between the sensor and the target. Since the interference wave is a sinusoidal wave, the sensor has high sensitivity when the target vibrates at around $0^{\circ}$ or $180^{\circ}$, or it has low sensitivity at around $\pm 90^{\circ}$. When the target vibrates with small amplitude of less than half-wavelength, the sensor has to detect the vibration at maximum sensitivity for accurate measurement. To reduce the measurement error, the sensor head is slowly moved by a microcomputer, and the output signal is automatically detected at maximum sensitivity.

\section{Experimental Arrangement}

A schematic of the experimental arrangement for detecting small vibrations is shown in Fig.4. The experimental arrangement consists of the sensor head with the LD and the
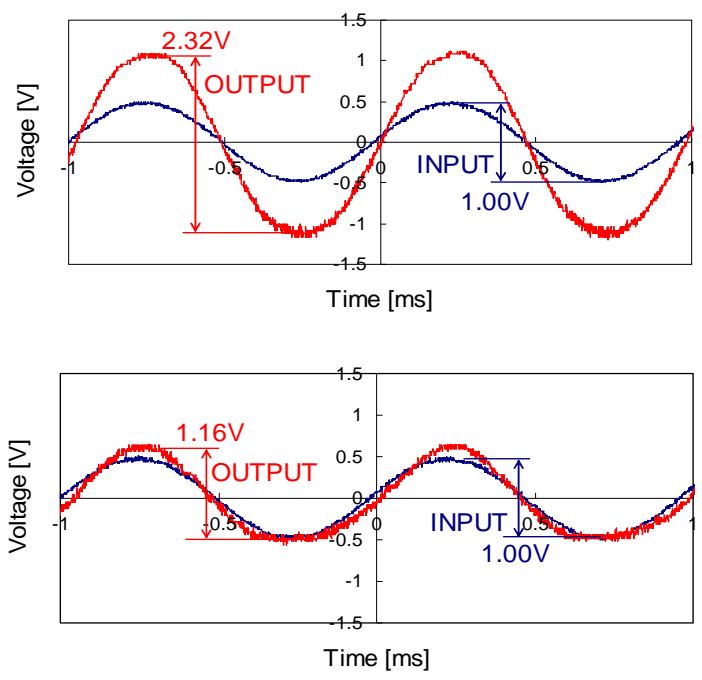

Fig.3 Two sensor output signals for different sensitivity

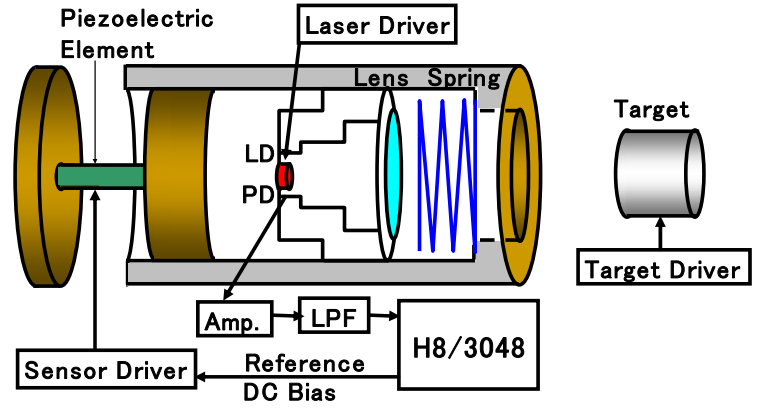

Fig.4 Block diagram of experiment

lens, the sensor driver for moving the sensor head, the target, the circuit for processing the output signal and the microcomputer. And the main unit with the sensor head is attached on an aluminum plate of $12 \mathrm{~cm}$ in length and $4 \mathrm{~cm}$ in width.

The LD in the sensor head is HL7859MG of HITACHI, and has a photodiode (PD) built into it. The LD has a wavelength of $780 \mathrm{~nm}$, an output power of $12 \mathrm{~mW}$ and a threshold current of $35 \mathrm{~mA}$. The lens with a focal length and a diameter of 10 $\mathrm{mm}$ is used. And these are put into an aluminium cylinder of $14 \mathrm{~mm}$ in diameter and $25 \mathrm{~mm}$ in length. The laser light is focused to $20 \mathrm{~mm}$ ahead on the target by the lens. The interference signal between the back-scattered light from the target and the output light of the LD is detected by the photodiode. To protect from the noise, a shielded wire from the output of the PD to the input of the amplifier is used.

The sensor head has a double cylinder structure, and the LD and the lens are put in an internal cylinder. The spring with a diameter of $10 \mathrm{~mm}$ and a length of $5 \mathrm{~mm}$ is set in front of the internal cylinder, and a piezoelectric element for the sensor driver is attached on the back. The piezoelectric element is a resin type AE0203D16 of TOKIN. The piezoelectric element is used for moving the sensor head, when the vibration amplitude is less than half-wavelength of the LD. And these are put into an external aluminum cylinder of $26 \mathrm{~mm}$ in diameter and $65 \mathrm{~mm}$ in length. The sensor head controlled by the microcomputer is quickly moved by a distance of more than half-wavelength of the $\mathrm{LD}$, and the reference signal is generated. In order to also get the maximum sensitivity of the sensor, the sensor head is slowly moved by the microcomputer.

The target is a metal type ASB170C801NP0 of TOKIN. To vibrate the target, the sinusoidal wave from a function generator is amplified by a power amplifier in the target driver circuit. The sinusoidal wave voltage with the bias voltage up to $13 \mathrm{~V}$ is applied to the target. The vibration frequencies of the target are changed from several $100 \mathrm{~Hz}$ to several $1000 \mathrm{~Hz}$, and the amplitude of the sinusoidal wave voltage is changed from several $100 \mathrm{mV}$ to several V.

The output signal from the PD is amplified by an amplifier, since the output signal is very small. The amplifier consists of transistors and operational amplifiers. The gain of the amplifier is about $50 \mathrm{~dB}$. At the first stage of the amplifier, a negative feedback amplifier with a field-effect transistor is used. The gain is about $20 \mathrm{~dB}$. At the second stage, an active low pass filter with operational amplifiers is used for eliminating the background noise. The active low pass filter passes all signals with a frequency lower than $10 \mathrm{kHz}$. At the 
third stage, an inverting amplifier with operational amplifiers is used. The gain is about $30 \mathrm{~dB}$. At the last stage, a limiter circuit with an operational amplifier and zener diodes is used. The microcomputer is damaged, when the voltage over $5 \mathrm{~V}$ is applied to it. The input voltage of the microcomputer is limited to $5 \mathrm{~V}$ or less by the limiter circuit.

The microcomputer is H8/3048 of Renesas Technology, and has an analog-digital conversion function, a digital-analog conversion function and many other functions. The clock frequency of the microcomputer is $16 \mathrm{MHz}$. And C language is used for the program. The microcomputer controls the sensor head to generate the reference signal by moving it by the distance of more than half-wavelength of the LD and to adjust the distance between the LD and the target automatically. The vibration amplitude and frequency of the target are calculated by the microcomputer.

\section{Flow of Measurement}

The flow chart of the vibration amplitude measurement by the microcomputer is shown in Fig.5. There are 2 main flows of the vibration amplitude measurement.

The first is the generation of the reference signal. The reference signal input data are converted from digital to analog by the microcomputer. The input data have a Gaussian profile. The analog voltage is applied to the piezoelectric element for the sensor driver. The output signal of the sensor generated by moving the sensor head is converted from analog to digital by the microcomputer. The output signal amplitude is evaluated from the output signal and is memorized every time. The maximum amplitude in the reference signal A is evaluated among the memorized values.

The second is the generation of the bias voltage signal. The bias voltage signal input data are converted from digital to analog by the microcomputer. And the analog voltage is applied to the piezoelectric element for the sensor driver. The output signal of the sensor generated by moving the sensor head slowly is converted from analog to digital by the microcomputer, and a larger amplitude value in the output signals is selected by comparing it with the previous one. The output signal amplitude at maximum sensitivity B is evaluated by repeating these in the predetermined numbers. The vibration amplitude of the target is calculated from two values of $\mathrm{A}$ and $\mathrm{B}$. The vibration frequency is calculated from the period between two successive peaks in the output signal.

\section{Results and Discussion}

The measured vibration amplitude as a function of the input voltage at the frequency of $1000 \mathrm{~Hz}$ is shown in Fig.6. The vibration amplitude of the target is proportional to the input voltage, and a small vibration of about $15 \mathrm{~nm}$ is able to be measured. This corresponds to about $1 / 50$ of the LD wavelength, and about $1 / 3$ of $50 \mathrm{~nm}$ measured by the manual operation. It becomes difficult to measure the small vibration under $15 \mathrm{~nm}$, since the signal and noise amplitudes are almost the same level.

The deviation in the vibration amplitude at $1000 \mathrm{~Hz}$ is shown in Fig.7. The vibration amplitude is measured 10 times, and the average of the vibration amplitude is calculated. The dispersion error of the vibration amplitude is evaluated from the average and the measured amplitude. The deviation is the ratio of the average of the dispersion error to the average of the

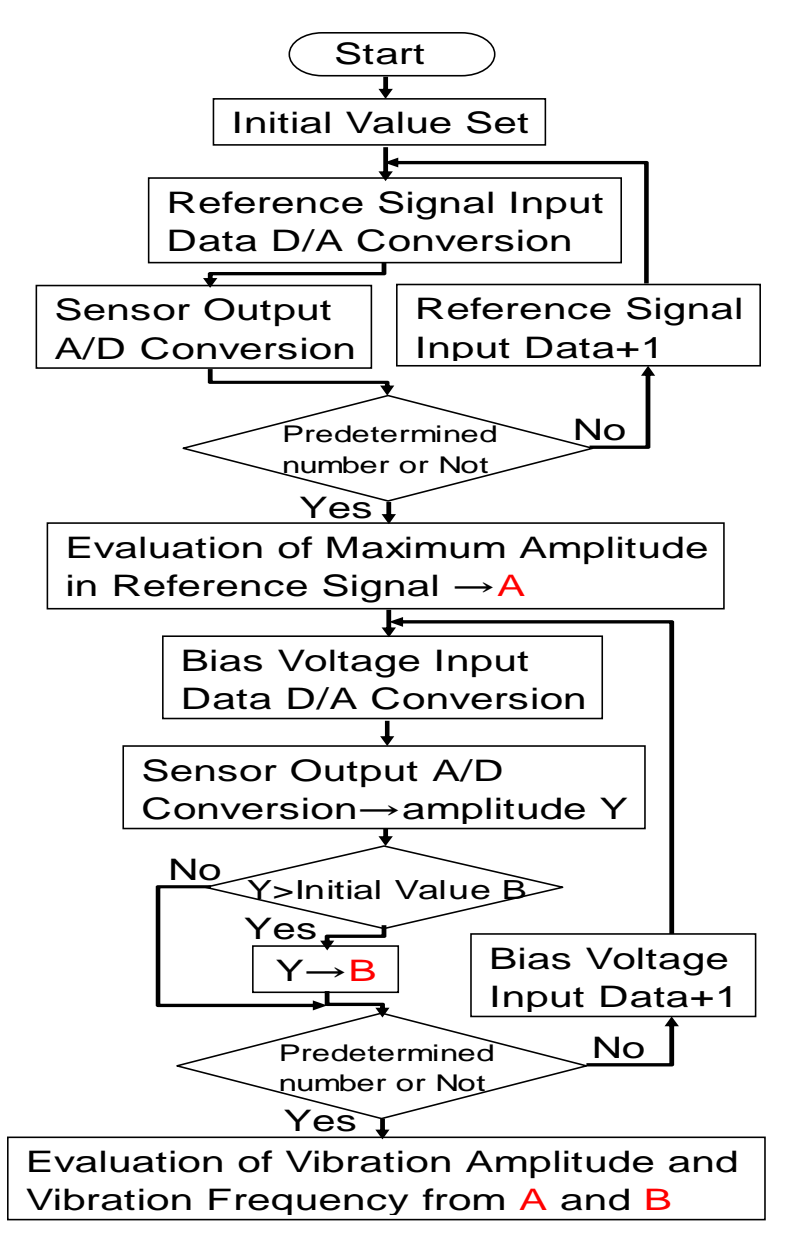

Fig.5 Flow chart of vibration measurement by microcomputer

vibration amplitude. The deviation of the vibration amplitude increases with decreasing the vibration amplitude, and is from 4 to $14 \%$. Because the noise seriously affects the output signal, when the vibration amplitude and hence the interference signal become small.

The frequency dependence of the small vibration sensor from $500 \mathrm{~Hz}$ to $3000 \mathrm{~Hz}$ is shown in Fig.8. The vibration amplitude is proportional to the input voltage at each frequency, and a small vibration of about $10 \mathrm{~nm}$ at $750 \mathrm{~Hz}$ and $3000 \mathrm{~Hz}$ can be measured. At $2000 \mathrm{~Hz}$ and $3000 \mathrm{~Hz}$, the vibration amplitude is less than that at other frequency. Because inertia may work on the target which is made of metal and has the mass of $43 \mathrm{~g}$. The deviation in the vibration amplitude from $500 \mathrm{~Hz}$ to $3000 \mathrm{~Hz}$ is the same tendency as one at $1000 \mathrm{~Hz}$, and is from 2 to $18 \%$.

The vibration frequency measured by the sensor is also proportional to the input frequency of the target between 500 $\mathrm{Hz}$ and $3000 \mathrm{~Hz}$. When the input frequency becomes less than $500 \mathrm{~Hz}$, the measured frequency is higher than input one. When the input frequency becomes more than $3000 \mathrm{~Hz}$, the measured frequency is lower than the input one.

When the vibration frequency becomes less than $500 \mathrm{~Hz}$, the deviation in the vibration amplitude increases, because the data loading time of the microcomputer is shorter than one period of the vibration. The sampling number of the 


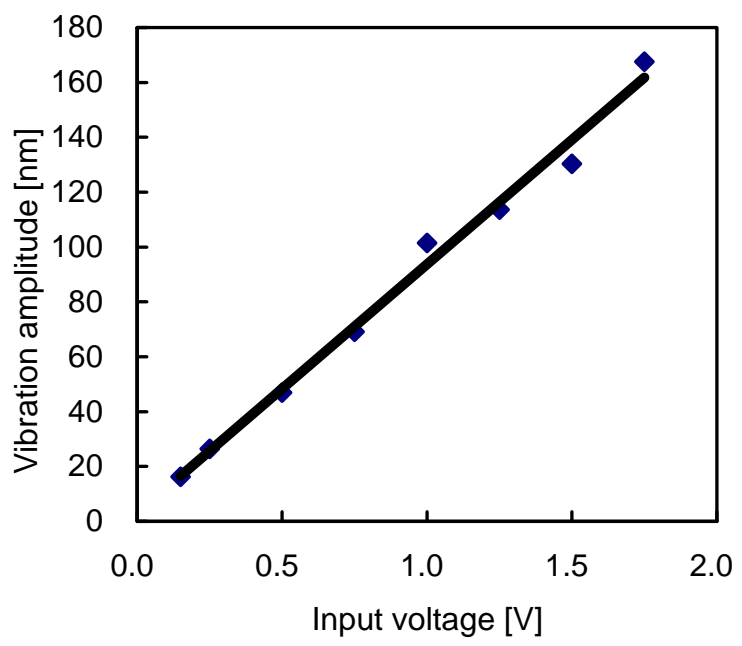

Fig.6 Vibration amplitude versus input voltage at $1000 \mathrm{~Hz}$

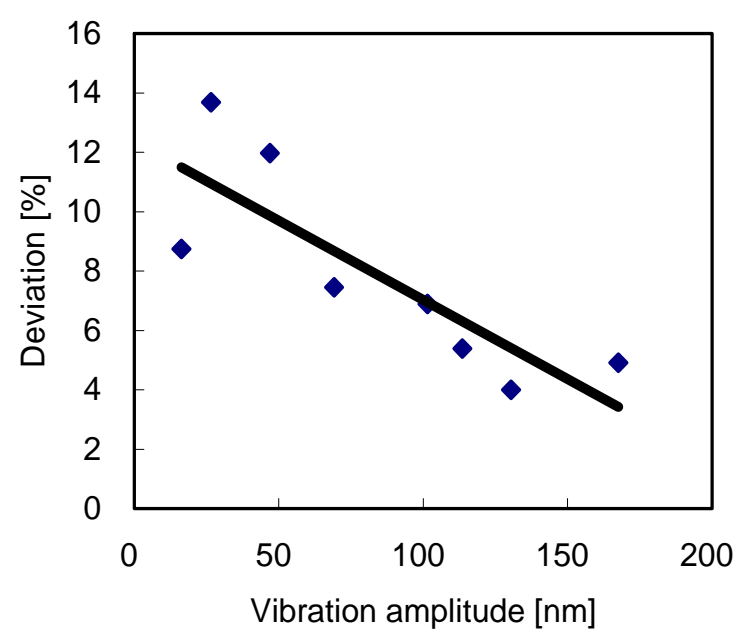

Fig.7 Deviation versus vibration amplitude at $1000 \mathrm{~Hz}$

microcomputer is 100 times. If the sampling number is increased, the vibration with a frequency of less than $500 \mathrm{~Hz}$ may be able to be correctly measured. When the vibration frequency becomes more than $3000 \mathrm{~Hz}$, the deviation in the vibration amplitude also increases, since the vibration frequency gets closer to the sampling frequency of the microcomputer. The sampling frequency of the analog-digital conversion function in the microcomputer is about $30 \mathrm{kHz}$. If the sampling frequency is increased, the vibration more than $3000 \mathrm{~Hz}$ may be able to be correctly measured.

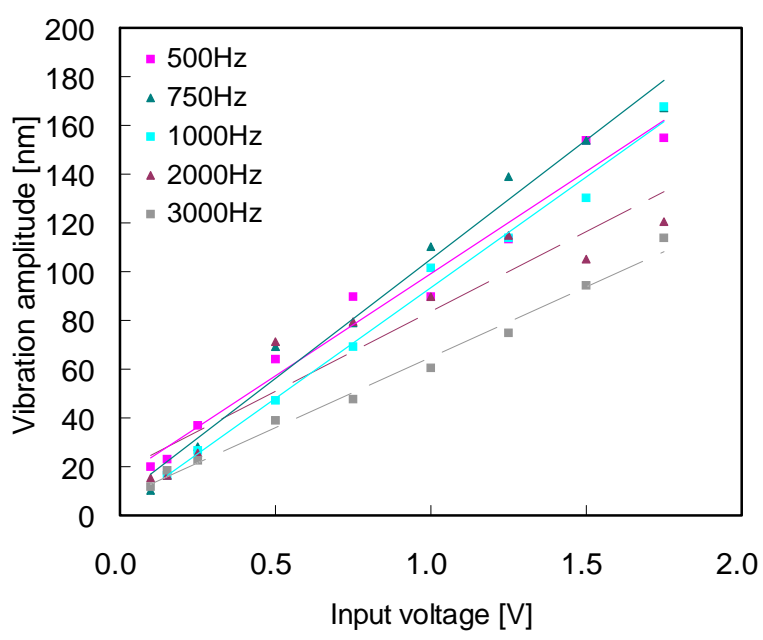

Fig.8 Frequency dependent sensor outputs

\section{Conclusion}

By making use of the self-coupling effect of the LD, we proposed a simple and compact vibration sensor, which had reference signal and could detect a small vibration without an external interferometer. A small vibration of about $10 \mathrm{~nm}$ was able to be measured. This corresponds to about $1 / 80$ of the LD wavelength. It was difficult to measure the small vibration under $10 \mathrm{~nm}$, since the signal amplitude of the sensor and the noise amplitude of the device were almost the same level.

The deviation in the vibration amplitude increased with decreasing the vibration amplitude, and was from about 2 to $18 \%$.

The small vibration was able to be measured without depending on the frequency, as long as the vibration frequency was in the range between $500 \mathrm{~Hz}$ and $3000 \mathrm{~Hz}$.

And also the small vibration frequency between $500 \mathrm{~Hz}$ and $3000 \mathrm{~Hz}$ was able to be measured.

\section{References}

1) P. J. Groot, G. M. Gallatin, and S. H. Macomber: Appl. Opt. 27(1988) 4475.

2) L. Krehut, J. Hast, E. Alarousu, and R. Myllyla: Opto-Electronics Rev. 11 (2003) 313.

3) G. Giuliani, S. Donati, and L. Monti: Proc. SPIE 4827 (2002) 353.

4) L. Scalise: Proc. SPIE 4827 (2002) 374.

5) Y. Nakao, N. Tsuda, and J. Yamada: T. IEE Japan, 121-C (2001) 1819.

6) A. Sakamoto, N. Tsuda, and J. Yamada: IEEJ Trans. EIS, 126 (2006) 1454. 